$>$ En 1918-19, la grippe « espagnole » a tué entre 2,5 et $5 \%$ de la population mondiale (entre 30 et 50 millions de morts). Image quintessenciée de l'épidémie moderne, presque tous les scénarios pandémiques s'accordent aujourd'hui pour y voir le modèle d'une pandémie «sévère». Peut-on toutefois sérieusement comparer le risque pandémique actuel à ce qui reste comme l'un des pires cataclysmes sanitaires de l'histoire? <

\section{Comme en 1918 ! \\ La grippe \\ « espagnole 》 \\ et nous}

Patrick Zylberman

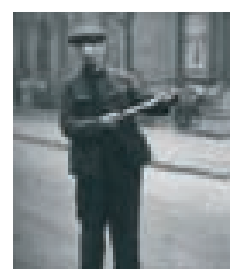

CERMES, Campus CNRS,

7, rue Guy-Môquet,

94801 Villejuif Cedex, France. zylberma@vjf.cnrs.fr
La pandémie de 1918 s'est singularisée à la fois par l'extrême pathogénicité de la souche $A(H I N)$, mais aussi par la tranche d'âge de ses victimes, les 20-35 ans, phénomènes qui ne se sont jamais répétés jusqu'ici. Un part importante de la mortalité est à mettre au compte des complications. En France comme ailleurs, la réponse a souffert d'une désorganisation extrême. Les moyens médicaux étant réquisitionnés par l'armée, la population civile a bien souvent manqué de tout: lits, médecins, infirmières, ambulances. En l'absence de traitement préventif ou curatif, les autorités ne pouvaient de toute façon que demeurer impuissantes à juguler la transmission grâce aux méthodes de santé publique (restrictions d'activités, isolement des malades). Plus que la guerre elle-même, les historiens accusent aujourd'hui la très forte croissance des communications par rail et par mer entre les cinq continents, entre le front et l'arrière, croissance encore accélérée par les hostilités. La multiplication des transports en tout sens a provoqué une sorte d' «égalisation bactérienne » entre catégories sociales et régions du monde. Un épisode singulier, dont les chances historiques de se reproduire dans les dix ans à venir sont somme toute très faibles.

La grippe prête aux prédictions les plus folles. Fin septembre 2005, le nouveau coordinateur pour la grippe aviaire et humaine à Genève n'avait pas hésité à prédire de 2 à 150 millions de morts dans le monde lors d'une prochaine pandémie. Quelques mois plus tôt, I'OMS citait le chiffre de 50 millions de décès: «comme en 1918 ! » De 30 à 50 millions de morts, les chiffres (approximatifs) auxquels s'arrêtent aujourd'hui les historiens de la grippe «espagnole» [1] représentent quatre à six fois le nombre de tués au combat lors de la guerre de 1914. La pandémie a tué entre 2,5 et $5 \%$ de la population mondiale, alors que les combats fauchaient environ $1 \%$ de la population dans les nations belligérantes. En 1918, la guerre a perdu (momentanément) la palme du massacre.

«Comme en 1918»: aucun des plans de réponse, aucun des scénarios qui ne prennent aujourd'hui pour «modèle» de la catastrophe annoncée la pandémie sévère de 1918, en effet. Ainsi pouvait-on voir en février dernier, à Boston, Anthony Fauci, le directeur du National Institute of Allergy and Infectious Diseases à Bethesda, brandir devant une assistance médusée, tel le mané thécel pharès tracé par la main sur le mur sous les yeux de Balthazar, une pancarte témoignant des horreurs de la grippe «espagnole» (Figure 1). De même, entre août 2004 et novembre 2005, le nombre des morts en sursis aux États-Unis a soudain décuplé (Tableau 1). L'administration américaine est passée de l'hypothèse d'une pandémie «modérée » à celle d'une pandémie «sévère »: «comme en 1918 » $[2,3]$ ! C'est d'ailleurs cette dernière hypothèse que retenaient aussi les experts français quelques mois auparavant [4]. La grippe de 1918 apparaît désormais comme l'image quintessenciée de l'épidémie moderne. Et ne vient-on pas de montrer tout récemment que le virus de 1918, que l'on croyait passé par le porc avant d'infecter l'homme, était lui aussi littéralement tombé du ciel $[5,6]^{1}$ ?

${ }^{1}$ Le séquençage du génome du type HIN1 de 1918 fait apparaître en effet que les trois polymérases (protéines basiques responsables de la réplication du virus) y sont identiques à celles des sous-types aviaires, $\mathrm{H} 5 \mathrm{~N} 1$ inclus; la souche de 1918 dériverait donc directement d'une souche aviaire, contrairement aux sous-types de 1957 et 1968 qui étaient, eux, des hybrides humains et aviaires. 
Ce n'est pas avant la seconde grande pandémie du $X X{ }^{e}$ siècle, la grippe «asiatique » de 1957, que la grippe « espagnole » est devenue de plein droit un objet d'histoire. En fait, depuis 1957, la grippe a changé de sens: non seulement l'on perçoit autrement, mais l'on perçoit autre chose. Le choix de l'hypothèse «sévère », «comme en 1918 », dévoile ce changement d'imagination historique. Ne sachant répondre par une invention pure à l'originalité des situations, ni décider sur la base d'un état de choses inédit, nous consultons ce que Paul Valéry appelait nos «souvenirs imaginaires », dont une illustration éloquente demeure la réaction caractéristique de l'administration US suite à l'apparition, début 1976, sur une base militaire du New Jersey, d'une poignée de cas de «grippe du porc». L'épidémie n'aura pas lieu, mais le virus était du même type $\mathrm{A}(\mathrm{HINl})$ que le virus de la grippe « espagnole » responsable de 500000 décès aux États-Unis en 1918-19, lequel n'avait plus circulé depuis 1920. Dans la presse, la réminiscence historique ne serait pas longue à refaire surface. Dans son mémo-

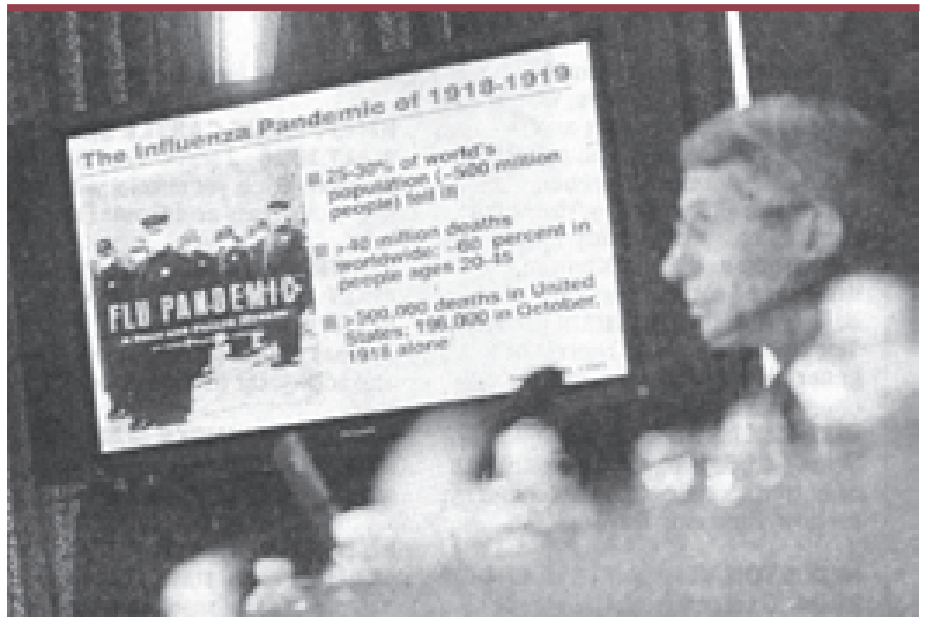

Figure 1. À droite du Dr A. Fauci, une photographie de 1918 montre des forces de police d'une grande ville américaine revêtues $d u$ masque protecteur. Le texte précise que le taux d'attaque se situait alors entre 25 et $30 \%$; plus de 40 millions de personnes dans le monde (dont $60 \%$ âgées de 20 à 45 ans) et 500000 Américains (dont 196000 pendant le seul moins d'octobre 1918) sont morts de la grippe (c) J. Carrier, Bloomberg News). randum adressé à la Maison Blanche, le directeur des CDC (Centers for Control Diseases) lui-même évoque la «possibilité d'une pandémie antigéniquement liée à celle de 1918 ». Parallèlement, le secrétaire à la Santé faisait adresser au président Gerald Ford un exemplaire d'Epidemic and Peace, 1918, d'Alfred Crosby - I'un des premiers ouvrages, sinon le premier, à traiter de la grippe « espagnole » en historien - paru au moment où éclataient les premiers cas [7], cependant que l'image de la pandémie hantait les discussions au Congrès [8]. Finalement, c'est sans études préalables mais poussé par les souvenirs d'un désastre antérieur que Washington prendrait la décision sans précédent de vacciner contre la grippe la population toute entière du pays. De 2006 à 1976, et de 1976 à 1918, la perception de la menace déroule ainsi à l'envers le film de la terreur épidémique au XXe siècle...

Le virus a débarqué en France, en avril 1918, avec le corps expéditionnaire américain. Venait-il du Middle-West, où l'infection avait été diagnostiquée début mars ? Étaitil originaire de Chine? La question n'est pas tranchée, mais le Midwest recueille aujourd'hui le plus grand nombre de suffrages. Après une première vague, dite «de printemps », une seconde vague, bien plus meurtrière, explosait à la fin du mois d'août à Brest, nouveau port de débarquement des troupes américaines. L'étincelle mettait alors le feu à toute la plaine, fauchant indifféremment soldats, ouvriers, généraux, femmes enceintes. Une troisième vague, moins létale, frappait ensuite au cours de I'hiver 1918-1919. Le taux de mortalité - près de $5 \%$ - représente cinq fois celui de la dernière pandémie du siècle précédent, la grippe «russe» de 1889-90. La grippe dite « espagnole », épithète injuste dont la raison tient peut-être au fait que, le pays n'étant pas en guerre, les nouvelles de la contagion n'étaient pas étouffées en Espagne comme ailleurs par la censure militaire, la grippe « espagnole » aura tué près de 2,3 millions de personnes $(4,8 \%)$ en Europe.

On sait que cette pandémie s'est singularisée par l'extrême pathogénicité du virus $\mathrm{A}(\mathrm{HINl})$ mais aussi par l'âge de ses victimes: les personnes âgées et les jeunes enfants ont été relativement épargnés (du moins par la vague d'automne), et ce sont les 20-35 ans qui ont supporté tout le poids de la morbidité et de la mortalité. Le phénomène ne s'est jamais reproduit depuis, sauf peut-être en 1977, au début de la pandémie [9]. Aussi la grippe se présente-t-elle d'abord comme un problème militaire. De mai 1918 à fin avril 1919 la mortalité dans l'Armée française 
s'élevait à 9,3\%o, la morbidité à $126 \%$. «La maladie attaque parfois un bataillon entier en une seule journée [10] ». Bien entendu, les gazés faisaient des cibles de choix. Trimballés d'un camp à l'autre, mal nourris, logés dans des casernements surpeuplés, les recrues de l'arrière seront cependant deux fois plus atteintes que les hommes montés en ligne; leur mortalité, même, trois fois supérieure. La grippe a pesé sur la tactique: intervenant lors des dernières grandes offensives, elle a éclairci les rangs, compliqué la mobilisation des renforts, désorganisé les transports.

Avec une mortalité de près de $4 \%$, la France a un peu moins souffert que l'Europe dans son ensemble. Le chiffre couramment avancé de 137000 décès civils est sans nul doute sous-estimé. D’abord tous les malades n'ont pas consulté ; ensuite, la grippe n'était pas une maladie à déclaration obligatoire ; enfin, les erreurs de diagnostic ont été légion. Nombre de décès sont dus aux surinfections bactériennes. À Toulon, par exemple, pleuropneumonies et pneumococcémies ont été fatales dans $10 \%$ des cas. Or ces complications ne figurent pas dans les statistiques sous le chef de la grippe. La censure de guerre ne facilite pas non plus le décompte des victimes. Une estimation plus récente aboutit au chiffre de 240000 décès civils et militaires pour l'automne de 1918 [11], estimation sans doute plus proche de la réalité (la Grande-Bretagne a enregistré 225000 décès, la Prusse 223000 ). En 1918, à Paris, la grippe occasionnait 48 décès en juillet et 45 en août - contre 2 durant la même période l'année précédente. De juin 1918 à avril 1919, elle contribuerait pour $18,5 \%$ à la mortalité générale dans la capitale; à Lyon, au même moment, elle entrerait pour $20 \%$ voire $40 \%$ dans l'augmentation de la mortalité (supérieure de 8,7\% à celle de 1913).

La désorganisation de la défense sanitaire serait extrême. L'armée a renvoyé les malades dans leurs foyers, contribuant à répandre la contagion. Or, en 1918, on compte 1 médecin pour 203 hommes d'effectifs dans les armées françaises (1 pour 376 dans les forces britanniques); comme $80 \%$ de ces praticiens sont issus de la réserve, il en résulte un grand désert médical dans la population civile. Le Havre, par exemple, comptait 90 médecins en 1914 : il n'en restait que 18 en 1917, soit un médecin pour 7500 habitants (1 pour 2300 en Grande-Bretagne, 1 pour 5700 en Allemagne). Crainte du ridicule, ce médecin se refuse d'ailleurs obstinément au port du masque [12]. Nous venons d'évoquer les médecins mobilisés: il en va de même des 200000 infirmières et infirmiers, au moment où les équipes soignantes, surmenées, sont décimées par la maladie. Les hôpitaux ont été réquisitionnés par les militaires. Fin septembre, l'Assistance publique ordonne de libérer des lits pour les civils: seul un quart des besoins est ainsi satisfait, et les malades ne sont pas isolés. Impossible de se procurer un flacon d'aspirine; plus d'alcool à $90^{\circ}$ sur les étagères des pharmacies; le glycérol, les corps gras sont réservés au Service des Poudres. À Paris, l'afflux est tel à la mi-octobre que l'accès aux salles devra être limité. La situation ne s'améliore qu'au début du mois de novembre alors que le nombre de malades va décroissant. Au fort de l'épidémie, les hôpitaux ont manqué de tout: lits, personnels, ambulances, médicaments.

Les services de l'État ne font pas meilleure figure. Malgré les appels réitérés d'Emile Roux, le directeur de l'Institut Pasteur, l'Académie des sciences semble figée dans une morne léthargie. Comme la maladie n'est pas déclarable, on ne peut consigner les malades à domicile; mais, manquant de personnel et de moyens de transport, les services d'hygiène se trouvent paralysés. L'Académie de médecine, le Conseil d'hygiène de la Seine ont bien demandé la fermeture des théâtres et autres lieux publics : en vain, les préfets n'appliquant pas ces mesures de peur de mécontenter la population. En plus de la guerre, la grippe a paralysé les services municipaux des grandes villes, pompes funèbres, service des eaux, enlèvement des ordures, ce qui n'a pu que compliquer encore un peu plus la défense sanitaire.

Et bien sûr, en l'absence de traitement préventif ou curatif, les autorités eussent été de toute façon impuissantes. II reste que cette désorganisation - ou inorganisation - des pouvoirs publics n'a peut-être pas été étrangère à la moindre résistance de la population, non sans doute à l'atteinte virale, mais tout au moins aux surinfections responsables d'un nombre important de décès.

Le scandale éclate au beau milieu de la vague létale, le 25 octobre 1918. Malmené à la Chambre, le soussecrétaire d'État à l'Intérieur (alors en charge de I'hygiène publique) se lamente: «Je suis appelé à répondre de la gestion de services qui ne sont pas placés sous mon autorité. » Les services de l'État en matière de santé se trouvaient en effet dispersés entre huit ministères! Vite, Clémenceau centralise le tout sous sa main, coup d'État bienfaisant dans lequel les partisans de la santé publique verront toujours le ministère de l'Hygiène idéal. Celui-ci-le premier du nom -est créé dans l'urgence et dans l'indigence le 21 janvier 1920. Comme en France, la pandémie a entraîné dans son sillage la création de ministères de la Santé un peu partout dans le monde, du Royaume-Uni aux Indes néerlandaises, et de la Nouvelle-Zélande à I'Union sud-africaine. Mais si l'urgence est parfois créatrice d'institutions, elle n'est pas gage de permanence. Créé sous les huées de parlementaires apeurés, en France le ministère de l'Hygiène sera bientôt dissous, par mesure d'économie, et ses bureaux fondus dans celui du Travail en 1924.

Les contemporains n'ont pas manqué d'accuser la guerre d'être responsable de cet holocauste dans l'holocauste. Qui, sinon l'universelle boucherie, aurait pu de la sorte transformer une maladie bénigne en une terrible catastrophe? Et cependant, les neutres comme les belligérants, Copenhague ou Stockholm comme Paris ou Berlin, ont été également frappés. Plus que sur la guerre elle-même, aujourd'hui les historiens jettent un œil de blâme sur la folle croissance des transports en tous sens, par rail ou par mer, croissance encore accélérée par les hostilités. Favorisant d'énormes rassemblements au front et à l'arrière dans des trains bondés de permissionnaires, multipliant les contacts 
entre les troupes et la population, la guerre a provoqué une sorte d' «égalisation bactérienne » entre catégories sociales, zones urbaines et rurales [13], faisant ainsi sauter les défenses contre la contagion, d'où peut-être la réputation «démocratique» de la grippe (en partie usurpée) qui, tout en réservant aux différents groupes d'âge des destins contrastés, n'en attaque pas moins assez uniformément les individus quelle que soit leur origine sociale [14]. Sillonnant les trois océans, des navires de tout tonnage ont porté la contagion sur tous les continents. Certaines populations d'Afrique subsaharienne, d'Amérique (Inuits et Indiens de l'Alaska) et d'Océanie (Maori) ont essuyé des mortalités effroyables (entre 27 et $80 \%$ ). Un cinquième de la population des Samoa occidentales (sous administration néozélandaise) a péri sous les assauts de la pandémie - une véritable décimation [15].

La dissémination d'un sous-type extrêmement pathogène $\mathrm{H} 5 \mathrm{Nl}$ dans la population animale, on l'a vu, conduit parfois à comparer la situation actuelle à celle de 1918. Semblable comparaison ne se peut. D'un côté, la vitesse décuplée des voyages intercontinentaux, la croissance exponentielle du nombre des voyageurs, le vieillissement des populations avec l'augmentation de la chronicité qui l'accompagne, les limites de nos capacités prédictives (composition antigénique du virus? pouvoir pathogène? c'est le noir absolu) sont sans nul doute des facteurs aggravants, sans compter les multiples interrogations relatives à la préparation des États et à leur réelle capacité de gestion d'une catastrophe de grande ampleur. D'un autre côté, la médecine est désormais en mesure de faire face (ainsi en 1957, quand le sous-type H2N2 de la grippe «asiatique » a pu être rapidement isolé, ou en 1968, lorsque des antibiotiques plus efficaces permirent de juguler les affections bactériennes opportunistes), alors qu'à la fin de la guerre de 1914, le virus de la grippe restait encore inconnu (il ne sera isolé qu'en 1933) ; la surveillance des virus circulant dans les populations humaines et animales mise en place au niveau international depuis dix ans est en outre capable de signaler sur-le-champ l'apparition de nouveaux cas et donc d'accélérer la mobilisation des pouvoirs publics; enfin, vaccins et antiviraux limiteront sans doute la mortalité, le nombre des hospitalisations et des malades et, par suite, la désorganisation des sociétés et des économies. Au total, donc, deux conjonctures qui n'ont que bien peu de choses en commun.

Depuis le début du XVIII ${ }^{e}$ siècle, on a compté entre dix et treize pandémies de grippe à la létalité plus proche de celle de 1957 et 1968 que de celle de 1918 qui semble plutôt unique dans sa sévérité [16]. En se fondant ainsi sur la fréquence des pandémies dans l'histoire, la probabilité qu'une grippe analogue à celle de 1918 intervienne dans les dix ans à venir n'excède peut-être pas $0,3 \%$ [17]. $\diamond$

\section{SUMMARY}

1918 lurks in everybody's mind. The «Spanish 》 flu and us

The 1918 pandemic is still unique in the history of flu pandemics. The pathogenicity of the virus was extreme, and young adults more than infants and old people were its main victims. Many a death was caused by complications. The response of the French authorities didn't live up to the emergency requirements. Hospitals being requisitioned by the military, the civilian population lacked everything: beds, doctors, nurses, ambulances, drugs. For want of preventive or curative medicine, authorities could have done very little at any rate: public health measures (quarantine and isolation of the sicks) were unable to stop contagion. More than the war itself, present day historians indict the war-boosted increase in railways and sea communications between the continents and between the rear and the front. This momentous growth in transportation activities brought about a «bacterial equalization » throughout social categories and regions of the world. A most singular episode, whose historical chances to replicate within the next ten years are rather slim. $\diamond$

\section{RÉFÉRENCES}

1. Johnson NPAS, Mueller J. Updating the accounts: global mortality of the 1918-1920 «Spanish » influenza pandemic. Bull History Med 2002 ; 76 : 105-15.

2. Meltzer MI, Cox NJ, Fukuda K. The economic impact of pandemic influenza in the United States: priorities for intervention. Emerg Infect Dis $1999 ; 5: 659-71$.

3. HHS Pandemic Influenza Plan. Washington: US Department of Health and Human Services, 2005.

4. Doyle A, Bonmarin I, Lévy-Bruhl D, et al. Estimation de l'impact d'une pandémie grippale et analyse de stratégies. Préparation à la lutte contre une pandémie grippale. Paris : Institut National de Veille Sanitaire, 2005.

5. Bubnoff AV. The 1918 flu virus is resurrected. Nature $2005 ; 437: 794-5$.

6. Kayser J. Resurrected influenza virus yields secrets of deadly 1918 pandemic. Science 2005 ; $310: 28-9$

7. Crosby AW. Epidemic and peace, 1918. Westport: Greenwood Press, 1976.

8. Neustadt RE, Fineberg HV. The swine flu affair. Decision-making on a slippery disease. Washington : US Department of Health, Education and Welfare, 1978.

9. Kilbourne $\varepsilon D$. A virologist's perspective on the 1918-19 pandemic. In: Phillips H, Killingray D, eds. The Spanish influenza pandemic of 1918-19. New Perspectives. Londres: Routledge, 2003: 29-38.

10. Zinsser H. Manifestations of influenza during the earlier periods of its appearance in France. Medical Record $1920 ; 97:$ 459-60.

11. Patterson KD, Pyle GF. The geography and mortality of the 1918 influenza pandemic. Bull History Med $1991 ; 65: 4-21$.

12. Hildreth ML. The influenza epidemic of 1918-1919 in France: contemporary concepts of aetiology, therapy, and prevention. Soc History Med $1991 ; 4$ : 277-94.

13. Delater. La grippe dans la nation armée de 1918 à 1921. Revue d'Hygiène 1923 ; $45:$ 409-634.

14. Zylberman P. A holocaust in a holocaust. The Great War and the 1918 Spanish influenza epidemic in France. In: Phillips H, Killingray D, eds. The Spanish influenza pandemic of 191819. New Perspectives. Londres : Routledge, 2003 : 191-201.

15. Rice G, Bryder L. Black November. The 1918 Influenza epidemic in New Zealand. Wellington: Allen and Unwin, $1988: 228$.

16. Patterson KD. Pandemic influenza 1700-1900: a study in historical epidemiology. Totowa NJ : Rowan and Littlefield, $1986: 91$.

17. A potential influenza pandemic: possible macroeconomic effects and policy issues. Washington DC : Congressional Budget Office, 8 décembre 2005 : 5-6.

\section{TIRÉS À PART}

P. Zylberman 